\title{
The hippocampal extracellular matrix regulates pain and memory after injury
}

\author{
Maral Tajerian ${ }^{1,2,3} \cdot$ Victor Hung ${ }^{2,3} \cdot$ Huy Nguyen ${ }^{2,3,4} \cdot$ Gail Lee $^{5} \cdot$ Lydia-Marie Joubert $^{6} \cdot$

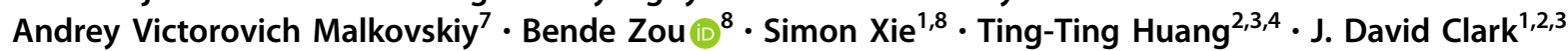

Received: 22 February 2018 / Revised: 26 May 2018 / Accepted: 18 June 2018 / Published online: 26 September 2018

(c) The Author(s) 2018. This article is published with open access

\begin{abstract}
Chronic pain poses a heavy burden for the individual and society, comprising personal suffering, comorbid psychiatric symptoms, cognitive decline, and disability. Treatment options are poor due in large part to pain centralization, where an initial injury can result in lasting CNS maladaptations. Hippocampal cellular plasticity in chronic pain has become a focus of study due to its roles in cognition, memory, and the experience of pain itself. However, the extracellular alterations that parallel and facilitate changes in hippocampal function have not been addressed to date. Here we show structural and biochemical plasticity in the hippocampal extracellular matrix (ECM) that is linked to behavioral, cellular, and synaptic changes in a mouse model of chronic pain. Specifically, we report deficits in working location memory that are associated with decreased hippocampal dendritic complexity, altered ECM microarchitecture, decreased ECM rigidity, and changes in the levels of key ECM components and enzymes, including increased levels of MMP8. We also report aberrations in longterm potentiation (LTP) and a loss of inhibitory interneuron perineuronal ECM nets, potentially accounting for the aberrations in LTP. Finally, we demonstrate that MMP8 is upregulated after injury and that its genetic downregulation normalizes the behavioral, electrophysiological, and extracellular alterations. By linking specific extracellular changes to the chronic pain phenotype, we provide a novel mechanistic understanding of pain centralization that provides new targets for the treatment of chronic pain.
\end{abstract}

Electronic supplementary material The online version of this article (https://doi.org/10.1038/s41380-018-0209-z) contains supplementary material, which is available to authorized users.

\section{Maral Tajerian}

maral@stanford.edu

1 Department of Anesthesiology, Stanford University School of Medicine, Stanford, CA, USA

2 Veterans Affairs Palo Alto Health Care System, Palo Alto, CA, USA

3 Palo Alto Veterans Institute for Research, Palo Alto, CA, USA

4 Department of Neurology and Neurological Sciences, Stanford University, Stanford, CA, USA

5 Department of Biology, Stanford University, Stanford, CA, USA

6 Cell Sciences Imaging Facility, Stanford University, Stanford, CA, USA

7 Department of Biomaterials and Advanced Drug Delivery, Stanford University, Stanford, CA, USA

8 AfaSci Research Laboratories, Mountainview, CA, USA

\section{Introduction}

Chronic pain (CP) is characterized by changes in nociception, affect, and cognition $[1,2]$ and is often resistant to classical treatment partly due to comorbid maladaptive plastic changes in the central nervous system (CNS) [3-7]. Specifically, a role for the hippocampus has been proposed owing to its role in cognition and memory as well as in modulating the overall pain experience. Functional, anatomical, and biochemical changes in the hippocampi of $\mathrm{CP}$ patients [8-10] and animal pain models $[7,11]$ have been reported. Despite interest in the role of hippocampal remodeling in $\mathrm{CP}$, experimental efforts thus far have been focused on cellular mechanisms of plasticity rather than the extracellular environment in which these cells function. This is likely due to both the neuro-centric nature of pain research and the technical difficulties of studying extracellular components. Here we show structural and biochemical alterations in the hippocampal extracellular matrix (ECM) that are linked to pain, cognitive dysfunction, and cellular plasticity in a mouse model of $\mathrm{CP}$. We report a 
constellation of deficits in working and location memory that are associated with decreased dendritic complexity, altered ECM microarchitecture, decreased ECM rigidity, and dysregulated ECM remodeling. We also report aberrant hippocampal long-term potentiation (LTP) and a reduction in specialized ECM nets around inhibitory interneurons, potentially accounting for the increased LTP. Finally, we show an amelioration of these maladaptive behavioral and physiological/biochemical phenotypes following an intervention to normalize ECM imbalance. These results delineate extracellular mechanisms of pain-related brain plasticity, thereby offering new therapeutic targets that could modulate already established CNS alterations present in $\mathrm{CP}$.

\section{Materials and methods}

\section{Animals}

Male C57BL/6J mice aged 12-14 weeks were purchased from a commercial supplier (Jackson Labs, Sacramento, CA, USA) and were allowed to habituate to the animal facility for a minimum of 10 days prior to the experiments. Mice were housed in groups of 4 on a 12-h light/dark cycle and an ambient temperature of $22 \pm 3{ }^{\circ} \mathrm{C}$, with food and water available ad libitum. All animal procedures and experimental designs were approved by the Veterans Affairs Palo Alto Health Care System Institutional Animal Care and Use Committee (Palo Alto, CA, USA) and followed the "animal subjects" guidelines of the International Association for the Study of Pain.

\section{Tibia fracture and cast immobilization}

Following the random allocation to the control or the fracture/cast group, mice were anesthetized with $1.5 \%$ isoflurane and underwent a distal tibial fracture in the right leg. Briefly, a hemostat was used to make a closed fracture of the right tibia just distal to the middle of the tibia and the hindlimb was wrapped in casting tape (cat. \#82001, Scotchcast $^{\mathrm{TM}}$ Plus, 3M, UK), as previously described [12]. After the procedure, the mice were given subcutaneous buprenorphine $(0.05 \mathrm{mg} / \mathrm{kg}])$ and enrofloxacin $(5 \mathrm{mg} / \mathrm{kg})$ for the next 2 days as well as normal saline $(1.5 \mathrm{ml}$, once) for postoperative analgesia, prevention of infection, and prevention of dehydration. Mice were inspected daily to ensure that the cast was positioned properly through the 3-week period of cast immobilization. Mice were provided with chow pellets postoperatively ad libitum; dietary gels were also made available on the cage floor for mice having undergone surgery. Casts were removed 3 weeks after surgery under brief isoflurane anesthesia. Naive, rather than sham (cast only), animals were used as controls since casting alone results in an intermediate phenotype where transient allodynia is observed both in rodents [13] and in humans [14].

\section{Behavioral testing}

All analysis was blinded to the identity and experimental condition of the animal. Mice were habituated to handling by the experimenter for a few minutes each day for 7 days before initiation of the behavioral tests.

\section{Mechanical sensitivity}

Calibrated monofilaments (Stoelting Co., USA) were applied to the plantar surface of the hind paw and the $50 \%$ threshold to withdraw ( $\mathrm{g}$ ) was calculated as previously described [15]. The stimulus intensity ranged from 0.004 to $1.7 \mathrm{~g}$, corresponding to filament numbers $1.65,2.36,2.44$, $2.83,3.22,3.61,3.84,4.08,4.17$, and 4.31. For each animal, the actual filaments used within the aforementioned series were determined based on the lowest filament to evoke a positive response (response $=$ flexion reflex) followed by five consecutive stimulations using the up-down method. The filament range and average interval were then incorporated along with the response pattern into each individual threshold calculation.

\section{Location memory test}

The arena was a square box $45 \times 45 \times 45 \mathrm{~cm}^{3}(L \times W \times H)$ made of white polyvinyl chloride. Four metallic enclosures (dimensions) were placed at each of the arena corners. Mice were placed into the arena from the middle of the south wall, with the north wall of the arena having a large visual cue $\left(28 \times 21.5 \mathrm{~cm}^{2}[W \times H]\right.$ sheet with alternating black and white columns [column width $=1.5 \mathrm{~cm}$ ]). Mice underwent one 10-min experience trial and a recall trial $24 \mathrm{~h}$ later. During the experience trial, one of the four metallic enclosures contained a female mouse (unfamiliar to the male mice). The female mouse was absent during the recall trial. Two identical sets of arenas were used for the two trials to ensure the absence of female odors during the recall trial. The total time spent investigating each of the four enclosures was recorded for the experience and recall trials. The percentage of time spent interacting with the enclosure with the female mouse was used as a measure of female interest (day 1) and female location memory (day 2).

\section{Y maze}

Y maze spontaneous alternation was used to assess rodents' tendency to explore new environments; they tend to explore 
a new arm of the maze rather than revisiting a previously entered arm. The Y-shaped testing arena was made of dark blue acrylics and consisted of 3 symmetrical arms (arms A, $\mathrm{B}$, and C) at $120^{\circ}$ angle with a dimension of $20 \times 8 \times 16 \mathrm{~cm}^{3}$ $(L \times W \times H)$ for each arm, each of which was decorated with different black and white visual cues at the end. Each mouse was introduced to the center of the maze and allowed to freely explore the three arms for $10 \mathrm{~min}$. Arm entriesscored when the animal reached the visual cue at the end of each arm-were recorded during the first $5 \mathrm{~min}$ of the test. Mice with good working spatial memory were expected to enter a new arm of the maze without immediate reentry to a previously visited arm. A unique triad combination of consecutive arm entries, i.e., $\mathrm{ABC}, \mathrm{BCA}, \mathrm{BAC}, \mathrm{ACB}$, $\mathrm{CBA}$, was used as a measurement of the spontaneous alteration. The percentage of alteration was calculated as (number of unique triad combination)/(total number arm entries-2).

\section{Dendritic labeling and analysis}

All analysis was blinded to the identity and experimental condition of the animal/tissue.

Mice received a single intracranial injection of adenoassociated virus (AAV) DJ-hSyn1 mCherry into the left hemisphere (to label neurons with red fluorescence protein) 7 weeks after injury according to a previously published protocol [16]. The injection coordinates were $\mathrm{AP}=-2 \mathrm{~mm}$ from bregma and $\mathrm{ML}=1.6 \mathrm{~mm}$ from midline to deliver AAV to the dentate gyrus (DG). The injection depth was $2.2 \mathrm{~mm}$ from the dura. A total of $0.5 \mu \mathrm{l}$ were injected at the rate of $0.1 \mu \mathrm{l} / \mathrm{min}$.

Animals were sacrificed $72 \mathrm{~h}$ after AAV injections. Tissue preparation was carried out as described in "Immunohistochemistry and histology." Two images per animal were randomly chosen for analysis. All neurons in the selected images were numbered and identified using the "Mark and Count" tool in Image J (Bethesda, USA). Then 10 IDs were randomly selected for further analysis in each $z$-stack image. Soma area was quantified using the Image $\mathrm{J}$ software. Dendritic length, number, and arborization were measured using manual tracing in Neuron $\mathbf{J}$ (Bethesda, USA). Sholl analysis was carried out using Fiji (Bethesda, USA) with the Sholl radius set at $5 \mu \mathrm{m}$.

\section{Scanning electron microscopy (SEM) and atomic force microscopy (AFM)}

All analysis was blinded to the identity and experimental condition of the animal/tissue. Following ketamine/xylazine anesthesia $(300 \mu \mathrm{l} / \mathrm{mouse})$ and transcardiac perfusion with $1 \times$ phosphate-buffered saline (PBS), hippocampi were dissected and decellularized per previously published methods
[17]. Decellularization was validated by the absence of DAPI (4,6-diamidino-2-phenylindole; nuclear marker) and $\mathrm{OsO}_{4}$ (lipid membrane marker) staining in samples chosen at random.

\section{Scanning electron microscopy}

Decellularized brain slices ( $2 \mathrm{~mm}$ thick) were fixed in $4 \%$ paraformaldehyde with $2 \%$ glutaraldehyde in $0.1 \mathrm{M}$ sodium cacodylate buffer (pH7.4) for $24 \mathrm{~h}$ at $4{ }^{\circ} \mathrm{C}$. Samples were then briefly rinsed in the same buffer before post-staining with $1 \% \mathrm{OsO}_{4}$ for $1 \mathrm{~h}$. OsO $\mathrm{O}_{4}$-treated samples were rinsed in water and gradually dehydrated in increasing concentrations of ethanol (50, 70, 90 100, 100\%, 10 min each). Samples were then stacked horizontally onto wire mesh dividers to keep them flattened and critically point dried with liquid $\mathrm{CO}_{2}$ using a Tousimis Autosamdri 815A apparatus and 10 min purge time (Tousimis, USA). Dried samples were mounted onto Aluminum SEM stubs using conductive copper tape and sputter-coated $(20 \AA$, Au/Pd) before imaging with a Zeiss Sigma FESEM using InLens SE detection at $3 \mathrm{kV}$ operating voltage (Carl Zeiss Microscopy Inc, USA). ECM abundance and fiber diameter were analyzed using Image J (Bethesda, USA) and microarchitectural motifs were analyzed using Mountainsmap (Digital Surf, France).

\section{Atomic force microscopy}

AFM force-distance measurements were carried out using a Park Systems NX-10 AFM in a liquid cell with deionized water. Tip sensitivity calibration was performed on glass surface after the experiments. The tip used for the experiments (NanoAndMore, $k=0.08 \mathrm{~N} / \mathrm{m}, R=1000 \mathrm{~nm}$ ) had a $\mathrm{SiO}_{2}$ sphere on its apex. The stiffness of tips from this batch was confirmed by the Sader method and not found to differ significantly from the nominal reported value. FDS curve data analysis was carried out using the commercially available SPIP software (Image Metrology, Denmark) and A/S and XEI software (Park Systems, Korea).

\section{Immunohistochemistry and histology}

All analysis was blinded to the identity and experimental condition of the animal/tissue. Mice were anesthetized by $300 \mu \mathrm{l}$ of ketamine/xylazine cocktail, followed by transcardiac perfusion with $1 \times$ PBS at 7 weeks after injury. Brains were carefully removed, post-fixed in $4 \%$ paraformaldehyde for 2 days, and placed in $30 \%$ sucrose solution. Brains were then embedded in agarose and crosssections were cut at room temperature at $40-\mu \mathrm{m}$ thickness on a vibratome (Leica vt $1200 S$ ). Ten sections/mouse were randomly chosen for staining. 


\section{Immunohistochemistry}

Free floating immunohistochemistry was done using trisbuffered saline $+0.1 \%$ tween-20 (TBST) as the wash buffer and 10\% donkey normal serum (cat. \#ab7475, Abcam, USA) in PBS as the blocking buffer. A permeabilization step was added (prior to blocking and staining) using $0.1 \%$ triton and $0.6 \%$ hydrogen peroxide in $1 \times$ TBST. The following primary antibodies/reagents were used (diluted in blocking buffer): rabbit polyclonal anti-mCherry (1:1000, cat. \#LS-C147181-30; Lifespan Biosciences, USA), rabbit polyclonal anti-parvalbumin (1:10,000; cat. \#PV25; Swant Inc., Switzerland), biotinylated Wisteria Floribunda Lectin (1:1000, cat. \#B-1355; Vector labs, USA), mouse monoclonal anti-GFP (anti-green fluorescent protein; 1:200, cat. \#ab184601; Abcam, USA), and rabbit polyclonal antiAggrecan (1:500, cat. \#AB1031; Millipore, USA). The following secondary antibodies were used (diluted in $1 \times$ TBST): Donkey anti-rabbit IgG $(\mathrm{H}+\mathrm{L})$ AlexaFluor 594 (1:500, cat. \#711-585-152; Jackson Immunoresearch, USA), Donkey anti-rabbit IgG $(\mathrm{H}+\mathrm{L})$ AlexaFluor 488 (1:500, cat. \#711-545-152; Jackson Immunoresearch, USA), Alexa Fluor ${ }^{\circledR} 555$ streptavidin (1:500, cat. \#S32355; ThermoFisher, USA), and Donkey anti-mouse IgG $(\mathrm{H}+\mathrm{L})$ AlexaFluor 488 (1:500, cat. \#715-545-150; Jackson Immunoresearch, USA). Sections were counterstained with DAPI and mounted on slides using fluoromount aqueous mounting medium (cat. \#F4680, Sigma, USA). Images were analyzed as a 2 - $\mu$ m-step $z$-stack of 20 slices $(\times 20$ objective magnification) using fluorescent imaging (Keyence BZX700, USA). Five sections per mouse were randomly chosen for analysis. $\mathrm{WFA}^{+}$and $\mathrm{PV}^{+}$cells were manually quantified in each $z$-stack using the "Count" tool and normalized to tissue area using Adobe Photoshop CS6. Matrix metalloproteinase 8 (MMP8) staining was quantified using the "Integrated Density" measure in Image $\mathrm{J}$ and normalized to tissue area (Bethesda, USA).

\section{Histology}

Analysis of overall matrix remodeling based on alterations in proteoglycan content was carried out using the multichromatic FAST (fast green, Alcian blue, Safranin O, and Tartrazine) staining method [18]. The staining sequence was as follows: distilled water-1 min, Alcian blue (strongly sulfated glycoproteins)-10 min, Safranin O (general detection of glycosaminoglycans) - $2.5 \mathrm{~min}, 50 \%$ ethanol-1 min, Tartrazine (mucin-associated ECM) - $10 \mathrm{~s}$, Fast green (glycoprotein-rich structures) -5 min. Slides were mounted using DPX mounting medium (cat. \#44581, Sigma, USA). Sections were imaged $(\times 10$ objective magnification) using brightfield microscopy (Keyence BZX700, USA). Five sections per mouse were randomly chosen for analysis. RGB values of each pixel of 40 images $(1440 \times 1920)$ were extracted. RGB values higher than $(250,250,250)$ were omitted from the extracted values in order to exclude white background color from the analysis. The RGB values were then converted to HSV values, which better represents the spectrum of human color perception. The converted HSV values were binned into 256 different ranges. Each bin count was divided by the total count of all HSV colors in each picture to acquire the relative proportion of each binned color with respect to all binned colors. All analysis was done using Matlab 2016.

\section{Analysis of ECM proteins}

All analysis was blinded to the identity and experimental condition of the animal/tissue. Mice were anesthetized (isoflurane) and sacrificed by decapitation at 7-8 weeks after injury. After the quick and careful removal from the skull, the hippocampus was extracted according to bregma coordinates and stored at $-80{ }^{\circ} \mathrm{C}$ until use.

\section{Two-color fluorescence western blotting}

Western blot analysis was performed according to standard procedures. Briefly, after sodium dodecyl sulfate-polyacrylamide gel electrophoresis and blotting, proteins on the membranes were detected by overnight incubation at $4{ }^{\circ} \mathrm{C}$ with the primary antibody followed by incubation with an infrared dye (IRDye)-conjugated secondary antibody. The following primary antibodies were used: rabbit monoclonal anti-Hapln1 (hyaluronan and proteoglycan link protein 1, 1:1 000, cat. \#ab181997; Abcam, USA), rabbit polyclonal anti-Aggrecan (1:500, cat. \#AB1031; Millipore, USA), rabbit polyclonal anti-HS2 (anti-hyaluronan synthase 2, 1:1000, cat. \#ab199794; Abcam, USA), mouse monoclonal anti-Neurocan (1:1000, cat. \#ab26003; Abcam, USA), mouse monoclonal antiBrevican (1:20, cat. \#73-281; Neuromab, USA), rabbit polyclonal anti-Versican (1:500, cat. \#AB1032; Millipore, USA), and mouse monoclonal anti-Chondroitin 4 Sulfate (1:1000, cat. \#MAB2030; Millipore, USA). The following secondary antibodies were used: IRDye $800 \mathrm{CW}$ goat antimouse IgG (H+L) (1:20,000; cat. \#926-32210; LI-COR Biosciences, USA) and IRDye 680RD goat anti-rabbit IgG (H+L) (1:20,000; cat. \#925-68071; LI-COR Biosciences, USA). $\beta$-Actin was used as an internal control and was detected with the mouse monoclonal anti- $\beta$-actin antibody (1:5000, cat. \#ab6276; Abcam, USA) followed by incubation with an IRDye $680 \mathrm{CW}$ goat anti-mouse $\operatorname{IgG}(\mathrm{H}+\mathrm{L})$ (1:20,000; cat. \#926-32220; LI-COR Biosciences, USA). The signals were detected using Odyssey (LI-COR Biosciences, USA) and quantified using the Image Studio software. 


\section{Enzyme-linked immunosorbent assay (ELISA)}

Levels of MMP2, 3, 8, and 9 were measured by multiplex ELISA (Eve Technologies, Canada). Tissue inhibitor of metalloprotease 1 (TIMP1) and TIMP2 levels were measured using commercially available kits per the manufacturer's instructions (cat. \#ab196265, \#ab100746, Abcam, USA)

\section{MMP8 downregulation}

All analysis was blinded to the identity and experimental condition of the animal/tissue.

We manipulated MMP generation capacity in hippocampal cells using short hairpin RNA (shRNA) administration through lentiviral delivery. Lentiviral particles for mouse MMP8-specific shRNA (MMP8 Mission 'shRNA; $1 \times 10^{6} \mathrm{TU} / \mathrm{mL}$; pLKO. 1 vector) or non-target shRNA control (Mission ${ }^{\circledR}$ pLKO.1 puro non-target shRNA control) were purchased commercially (Sigma Aldrich) and used to downregulate MMP levels in the hippocampus. The sequence for MMP8-specific shRNA is: CCGGGCCTTGATGTACCCAAACTATCTCGAGATAGTTTGGGTACATCAAGGCTTTTTG. Mice received single intracranial injections of lentivirus into each of the left and right hemispheres 3 weeks after injury. The injection coordinates were $\mathrm{AP}=-2 \mathrm{~mm}$ from bregma and $\mathrm{ML}$ $= \pm 1.6 \mathrm{~mm}$ from midline to deliver the lentivirus to the DG. The injection depth was $2.2 \mathrm{~mm}$ from the dura. A total of $1 \mu \mathrm{l}$ was injected in each hippocampus at the rate of 0.1 $\mu \mathrm{l} / \mathrm{min}$. To visualize lentiviral spread, a pLKO.1-CMVtGFP vector with a non-target SHC016 shRNA sequence (Sigma Aldrich, USA) was used as described above.

\section{Electrophysiology}

All analyses were blinded to the identity and experimental condition of the animal/tissue.

Following deep anesthesia (isoflurane) and sacrifice, the brain was quickly removed into ice-cold artificial cerebrospinal fluid (ACSF) bubbled with $5 \% \quad \mathrm{CO}_{2} / 95 \% \mathrm{O}_{2}$ continuously. The ACSF was constituted as (in $\mathrm{mM}$ ) $\mathrm{NaCl}$ 131.0, $\mathrm{KCl} 2.5, \mathrm{KH}_{2} \mathrm{PO}_{4} 1.2, \mathrm{CaCl}_{2} 2.4, \mathrm{MgSO}_{4} 1.3$, $\mathrm{NaHCO}_{3}$ 26.0, and glucose 10.0 (pH 7.4). Right hippocampus was cut into slices $(400 \mu \mathrm{m}$ thick) with a tissue slicer (Stoelting Co., IL) and incubated in room temperature with ACSF oxygenated continuously for at least $1 \mathrm{~h}$. Extracellular recording was made in a Haas chamber (Harvard Apparatus, USA) with submerged mode at room temperature. Slices were continuously perfused with ACSF bubbling with $5 \% \quad \mathrm{CO}_{2} / 95 \% \mathrm{O}_{2}$ at flow rate about $1.5 \mathrm{ml} / \mathrm{min}$ with a peristaltic pump (Buchler, USA).
The recording electrode was made with borosilicate glass (Warner Instruments) and filled with regular ACSF (resistance $=\sim 1-3 \mathrm{M} \Omega)$. Biphasic current stimulating pulses $(0.4$ $\mathrm{ms})$ were delivered with an interval of $10 \mathrm{~s}$ through concentric bipolar electrode (CBARC75, FHC). To record field population spikes in the dorsal DG, the recording electrode was placed at the lateral part of upper granular cell layer and stimulating electrode was placed immediately above hippocampal fissure to tease the bypassing perforant pathway fibers. Input/output curves were obtained for each slice with stimulus intensities ranging from threshold to $1.0 \mathrm{~mA}$. Baseline was recorded with stimulus intensity evoking half of maximal response. High frequency stimulation consisted of $2 \times 100$ pulse trains $(1 \mathrm{~s}, 100 \mathrm{~Hz})$ in baseline stimulus intensity. Slices were recorded within $6 \mathrm{~h}$ after dissection. Data were acquired with an Axopatch 2B amplifier through Digitizer 1320A using ClampEx 10.4 (Molecular Devices, USA).

The amplitude and slope of population spikes were measured from the initial phase of negative wave with Clampfit10.4 (Molecular Devices, USA). Each data point was measured as an average of three consecutive traces. LTP following high frequency stimulation was plotted as a percentage of the baseline. Representative traces are the average of 6 consecutive stimulations in a 1-min period. Paired pulse ratio (PPR) was recorded with baseline stimulus intensity in $50 \mathrm{~ms}$ inter-pulse interval. PPR was calculated as a percentage of the difference between the two responses divided by the first one.

\section{Statistical analysis}

All data are expressed as mean \pm s.e.m. (standard error of mean). Analysis of repeated parametric measures was accomplished using a two-way analysis of variance (ANOVA) followed by Holm-Sidak post hoc test for multiple comparisons. When comparing three groups, a one-way ANOVA was used followed by Holm-Sidak post hoc test for multiple comparisons. For simple comparisons of two groups, two-tailed Student's $t$ test was used. Welch's correction was used when the assumption of equal variances was not met. Significance was set at $P$ value $<0.05$ (Prism 5; GraphPad Software, USA). Sample size for each experimental end point is indicated in the figures.

\section{Results and Discussion}

We used a mouse model of $\mathrm{CP}$ due to tibia fracture to demonstrate, 7 weeks following injury (Fig. 1a), behavioral signs of tactile allodynia indicated by reduced mechanical thresholds on the injured hindpaw (Fig. 1b). Even though the tibia fracture itself had healed, at this chronic time point, 


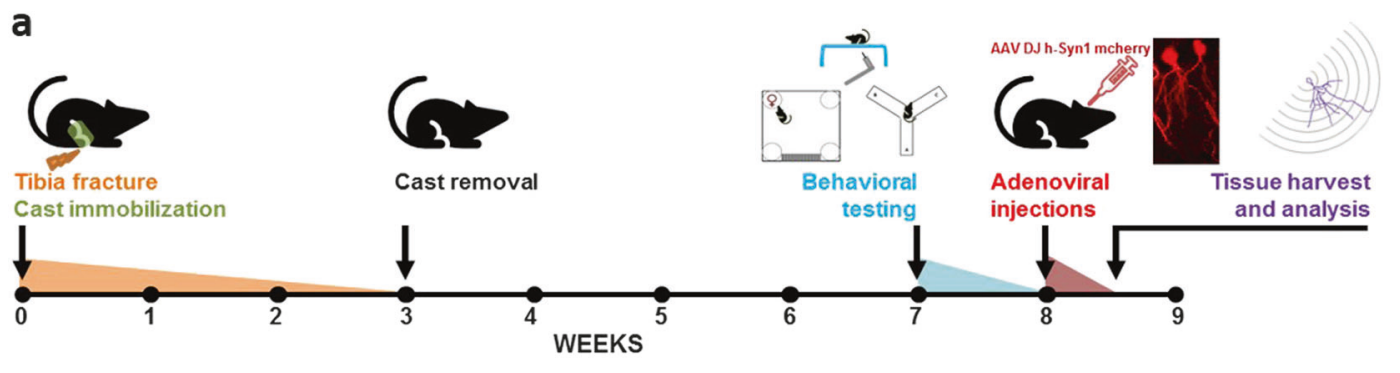

b

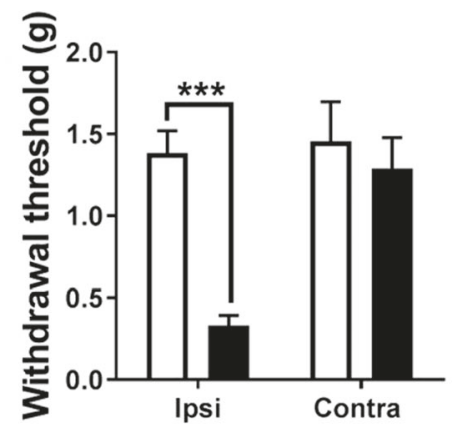

e

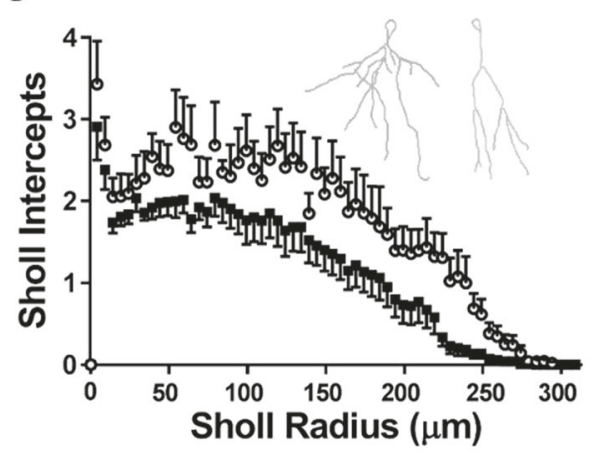

Fig. 1 Alterations in hippocampal cytoarchitecture parallel behavioral signs of pain and memory dysfunction following peripheral injury. a Experimental timeline: Male C57BL/6 mice underwent tibia fracture and cast immobilization. All analysis was carried out during the 7-9week period. b Seven weeks following peripheral injury, mice display a reduction in mechanical thresholds on the ipsilateral hindpaw (twoway ANOVA, post hoc Holm-Sidak test for multiple comparisons, $n$ $=16-17$ mice/group). $\mathbf{c}$ Injured mice are deficient in location memory recall 1 day following exposure to a female mouse (two-way ANOVA, post hoc Bonferroni test for multiple comparisons, $n=7-9$ mice/ d
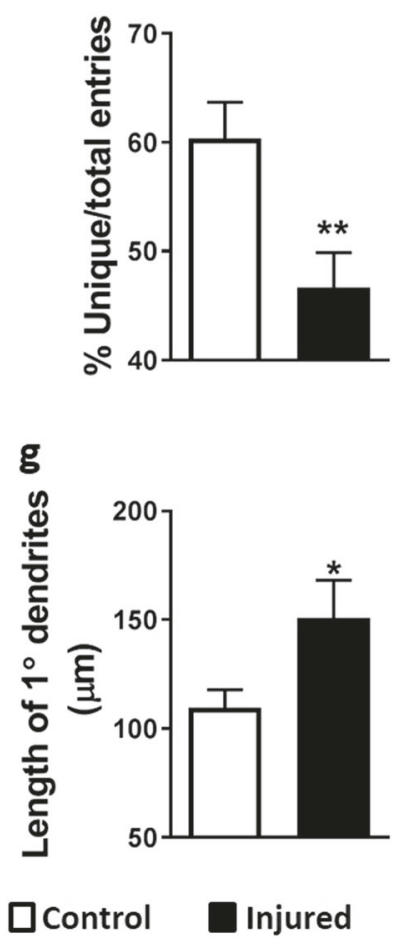

group). d Similarly, they demonstrate deficits in working spatial memory in the Y maze as evidenced by the percentage of unique triad combination of consecutive arm entries (Student's $t$ test, $n=8-9$ mice/ group). e-g AAV DJ-hSyn1 mCherry was stereotaxically injected into the hippocampus to fluorescently label a small percentage of neurons. Representative neuronal tracings are included in the inset (left = Control, right $=$ Injured). Sholl analysis revealed decreased dendritic complexity following injury, in addition to increased length of primary dendrites. (Student's $t$ test, $n=10-15$ mice/group). Error bars are s.e. m. $* P<0.05 ; * * P<0.01 ; * * * P<0.005$

increased dendritic length (Fig. 1g) in the injured group. These observations reflect hippocampal plasticity that persists long after the initial injury to the limb, and considering that pain encompasses affective-motivational and cognitiveevaluative dimensions, these signs of hippocampal plasticity not only parallel the memory deficits observed in $\mathrm{CP}$ but also further bolster the role of the hippocampus in pain processing [21]. These findings complement previous reports of pain-related structural and functional changes in the hippocampus: compared to control subjects, patients 


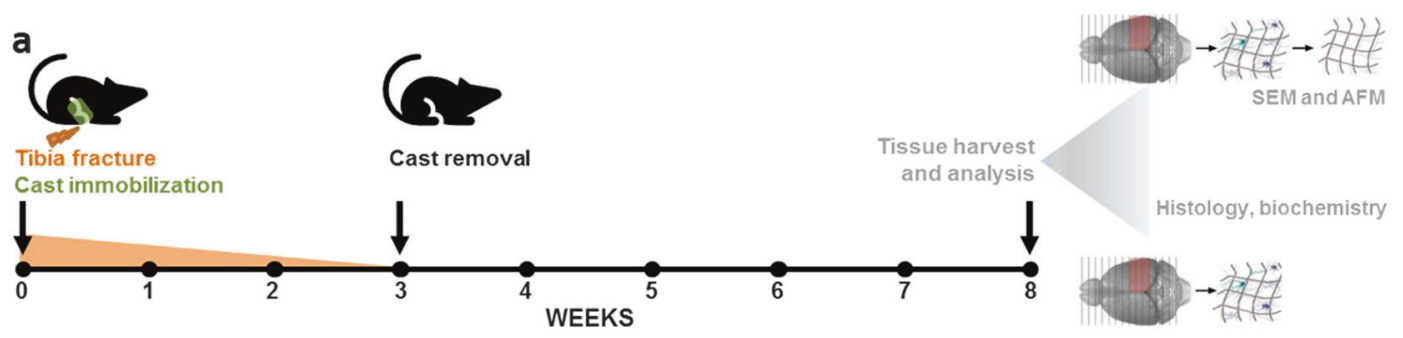

b
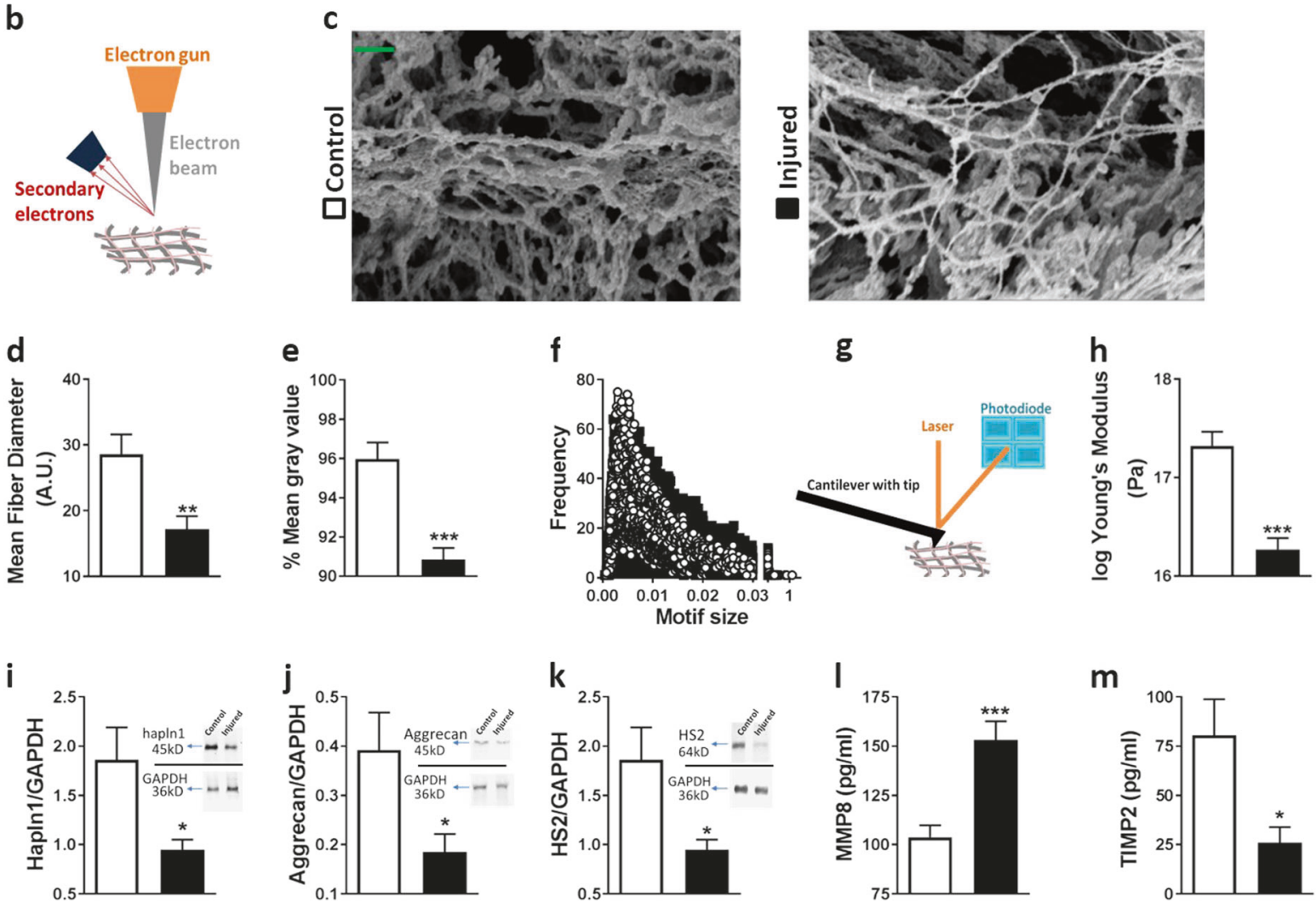

Fig. 2 Peripheral injury is accompanied by biophysical and biochemical alterations in the hippocampal extracellular matrix. a Experimental timeline: Male C57BL/6 mice underwent tibia fracture and cast immobilization. Seven weeks following injury, brains were decellularized for scanning electron microscopy (SEM) and atomic force microscopy (AFM) analysis or left intact for histological and biochemical analyses. b-f Surface scanning electron micrographs demonstrated changes in ECM microarchitecture in the form of altered geometrical micromotifs, and reduced mean fiber diameter as well as a

with CP demonstrated increased hippocampal gray volume [8] and increased hippocampal-prefrontal cortex connectivity $[9,10]$. Similarly, changes in hippocampal volume were observed in preclinical models of pain [11]. Finally, we have reported biochemical changes in the form of decreased hippocampal levels of synaptophysin and brainderived neurotrophic factor in mice with peripheral injury [7].

Since the advent of the neuron doctrine [22], the majority of brain plasticity studies have focused on neurons, with a more recent interest in glia. However, it is well known that reduction in mean gray values. Scale bar $=400 \mathrm{~nm}$. (Student's $t$ test, $n$ $=5$ mice/group). $\mathbf{g}, \mathbf{h}$ The examination of ECM stiffness using contact-mode AFM revealed decreased ECM rigidity in the hippocampi of injured mice (Student's $t$ test, $n=22$ measurements each taken from 5 mice/group). $\mathbf{i}-\mathbf{m}$ Injury is associated with decreased levels of hapln1, aggrecan, and HS2, in addition to decreased levels of MMP8 and increased levels of TIMP2 (Student's $t$ test, $n=8-12$ mice/group). Error bars are s.e.m. $* P<0.05$; $* * P<0.01 ; * * * P<0.005$

the ECM in the CNS is crucial in the regulation and stabilization of plasticity, including synaptic activity, both during development and in adulthood [23]. Thus, a flexible response by the ECM is necessary to permit and/or facilitate cellular alterations. To understand whether global ECM changes in the hippocampus occur in response to pain, we isolated the ECM by decellularizing the dissected hippocampi (Fig. 2a), and measured structural changes using surface SEM and contact-mode AFM. Injured animals displayed altered ECM microarchitecture in the form of thinner and less abundant ECM fiber as well as alterations in 
geometrical motifs compared to controls (Fig. 2b-f). Furthermore, injury was associated with reduced stiffness of the hippocampal ECM, evidenced from the change in Young's modulus (Fig. 2g, h) measured by force-distance AFM indentation measurements. Our findings of decreased ECM stiffness and decreased dendritic complexity complement studies where rigid ECM substrates were shown to favor increased dendritic numbers and branching while a softer ECM was linked to fewer and longer neurites and an increased number of synapses $[24,25]$.

Our biophysical measures support the hypothesis that, in the adult brain, peripheral injury is linked to significant extracellular alterations of the ECM at the structural level. What, then, are the individual ECM components involved? The brain ECM that occupies $20 \%$ of adult brain volume is mainly composed of proteoglycan and hyaluronan components [26] and is synthesized by neuronal, glial, and endothelial cells. Based on our AFM differences in structural rigidity, we focused on structural-as opposed to matricellular-ECM components. We first stained the tissue for proteoglycans based on their electric charge using the multichromatic FAST (see Methods). Our results show qualitative differences between the groups, where safranin $\mathrm{O}$ staining appears to be reduced after injury, indicating diminishing glycosaminoglycan content (Supplementary Fig. 1). Next, we quantified biochemically both the various components of the ECM and ECM-modulating enzymes. We found decreased levels of hapln1 and aggrecan (Fig. 2i, $\mathrm{j}$ ), two key components of the specialized network of ECM known as the perineuronal net (PNN), in addition to decreased levels of HS2 (Fig. 2k), an enzyme that synthesizes hyaluronic acid, the backbone of the ECM. This profile is similar to that observed in the hippocampus during status epilepticus, where enhanced neuronal activity is associated with decreased aggrecan, hapln1, and HS3 [27]. Additionally, we show increased levels of MMP8 (Fig. 21), an aggrecan-degrading metalloprotease, and decreased levels of TIMP2 (Fig. 2m). MMPs are well known as plasticity stimulators and mediators of synaptic physiology and neurite outgrowth. Furthermore, MM8 inhibition protects against systemic inflammation [28] and its upregulation in CSF can serve as a biomarker of spinal cord injury [29]. More recently, MMP8 was shown to act as a neuroinflammatory mediator in activated microglia [30] and to play a critical role in brain damage following ischemic injury [31]. TIMP2 is an endogenous protein that reversibly inhibits a wide range of MMPs - including MMP8-in a 1:1 stoichiometric manner [32], and a disturbed balance between TIMPs, MMPs, and ECM proteins is often seen in various neurodegenerative pathologies [33, 34]. More specifically, a recent study has shown that TIMP2 improves hippocampal cognition in aged mice [35]. No changes were observed in the abundance of neurocan, brevican, versican, chondroitin 4 sulfate, MMP2, MMP3, pro-MMP9, and TIMP1 (Supplementary Fig. 2).

Since our data suggest an altered state of homeostatic ECM plasticity after peripheral injury, we next tested whether the restoration/prevention of ECM dysregulation could result in the amelioration of the nociceptive and cognitive changes observed in CP. Our target of choice was MMP8 instead of TIMP2 due to specificity, since it was the only MMP that was upregulated after injury and since it can degrade hapln1 and aggrecan, two ECM components that were downregulated in our model. To assess the functional role of MMP8 in contributing to the broader CP phenotype, we downregulated MMP8 levels in bilateral hippocampi using lentiviral-delivered shRNA (Fig. 3a-c). This decrease in hippocampal MMP8 resulted in improvements in tactile allodynia in the ipsilateral but not in the contralateral hindpaw (Fig. 3d, e) as well as improvements in long-term memory in the female location memory assay (Fig. 3f) and working memory in the Y maze (Fig. 3g). To our knowledge, this is the first report linking MMP8 to hippocampal and behavioral plasticity.

Having demonstrated both neuronal and extracellular alterations in the hippocampus in $\mathrm{CP}$, we aimed to delineate the mechanistic link between the two. Electrophysiological analysis of LTP in the dorsal DG showed an aberrant increase in field excitatory postsynaptic potential in $\mathrm{CP}$ mice that was absent in the shRNA-treated mice (Fig. 4b-d). These data complement findings from animal studies where chronic visceral pain was shown to be linked to enhanced hippocampal LTP [36] as well as human studies where a widespread increase in hippocampal connectivity was observed in patients with chronic low back pain [10]. No changes in input/output or PPR measures were noted, suggesting that injury is not linked to decreased synaptic transmission efficiency or decreased probability of neurotransmitter release (Supplementary Fig. 3).

Unlike LTP in immature brains, the post-injury aberrant LTP levels in an adult hippocampal granular cell layer following high frequency stimulation implies a need for reduced GABA-ergic inhibition [37]. Indeed, recent studies have shown a role for GABA-ergic signaling in modulating synaptic plasticity in the adult brain [38] as well as evidence for parvalbumin-positive interneurons regulating hippocampal LTP [39]. Since many hippocampal inhibitory interneurons are surrounded by a PNN, and since GABAergic transmission and PNN alterations are classically viewed as "plasticity brakes" [40], we therefore hypothesized that a possible mechanism by which ECM can alter neuronal physiology is through the stabilization of inhibitory GABA-ergic interneurons in the hippocampus through the PNN that surrounds their cell bodies and proximal neurites. Chondroitin sulfate proteoglycans, including aggrecans, are main constituents of the PNN 
a

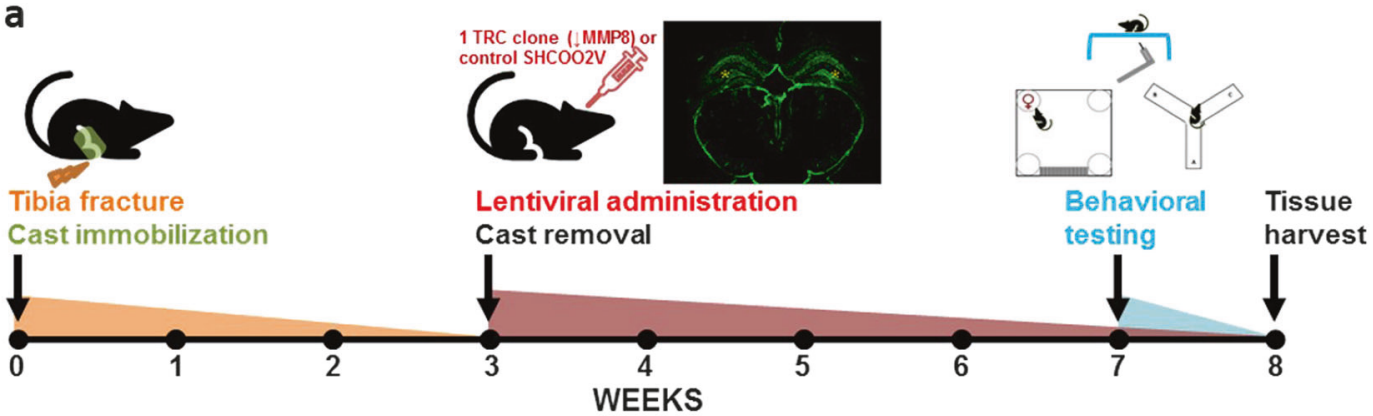

b

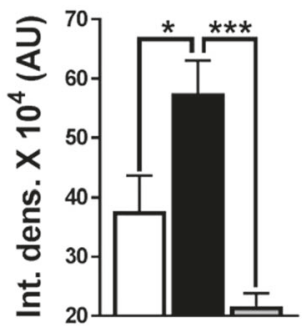

d

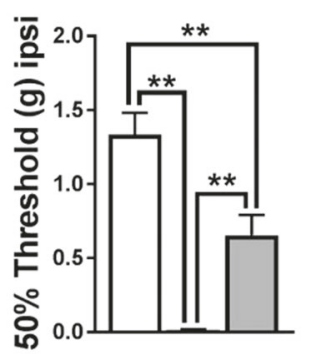

C

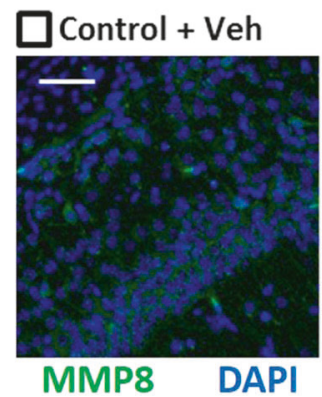

e

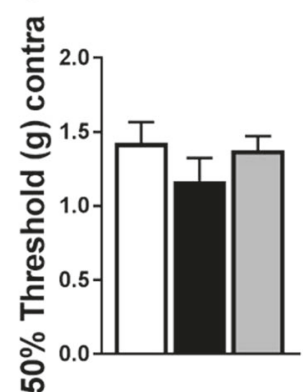

f
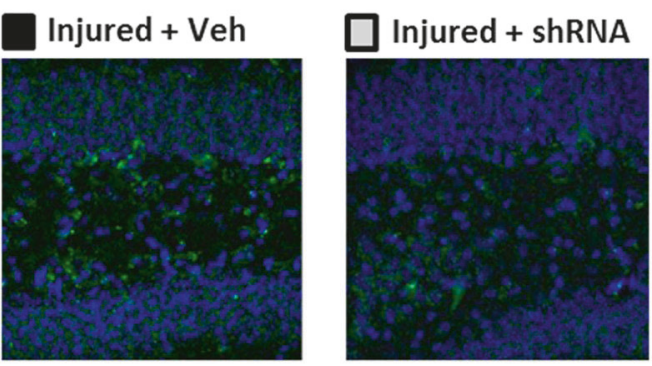

g
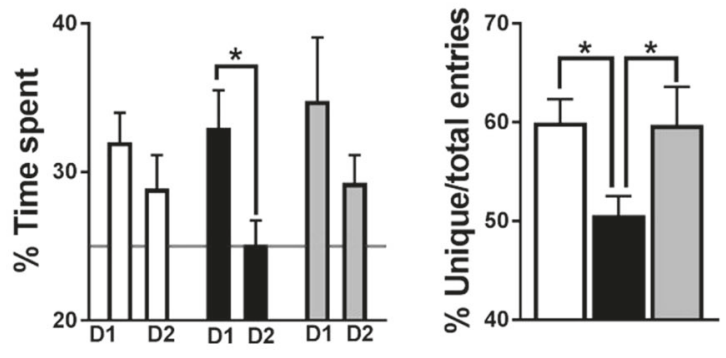

Fig. 3 a Experimental timeline: Male C57BL/6 mice underwent tibia fracture and cast immobilization. Three weeks following injury, $\downarrow$ MMP8 or control shRNA was injected in bilateral hippocampi (inset shows fluorescent GFP labeling of lentiviral spread, yellow asterisks indicate the site of injection). Behavioral and immunohistochemical analyses were carried out 7-8 weeks after injury. b, c Upregulated MMP8 levels in injured mice were normalized following shRNA treatment (one-way ANOVA, post hoc Holm-Sidak test for multiple comparisons, $n=10-12$ mice/group). Scale bar $=100 \mu \mathrm{m}$. d-g MMP8 downregulation ameliorates signs of mechanical allodynia in the ipsilateral hindpaw (no differences were seen in the contralateral hindpaw) as well as memory dysfunction (female location memory and Y maze) following injury (one-way $\operatorname{ANOVA}(\mathbf{d}, \mathbf{f})$ or two-way ANOVA (e), post hoc Holm-Sidak test for multiple comparisons, $n=$ 9-16 mice/group). Error bars are s.e.m. $* \mathrm{P}<0.05 ; * * * P<0.005$

Despite mounting evidence for the role of hippocampal remodeling after an injury to the periphery and $\mathrm{CP}$, the adult hippocampal ECM is often erroneously thought to be static. Our results show that the sustained anatomical, physiological, and biochemical dysregulation of the hippocampal DG ECM after peripheral injury supports pain as well as cognitive deficits characteristic of pain patients. Therapies directed at controlling maladaptive ECM plasticity may constitute a novel approach to controlling $\mathrm{CP}$ and its undesirable sequelae, going beyond examining nociceptive pathways themselves, and addressing structural factors supporting the broader pain experience. 
a
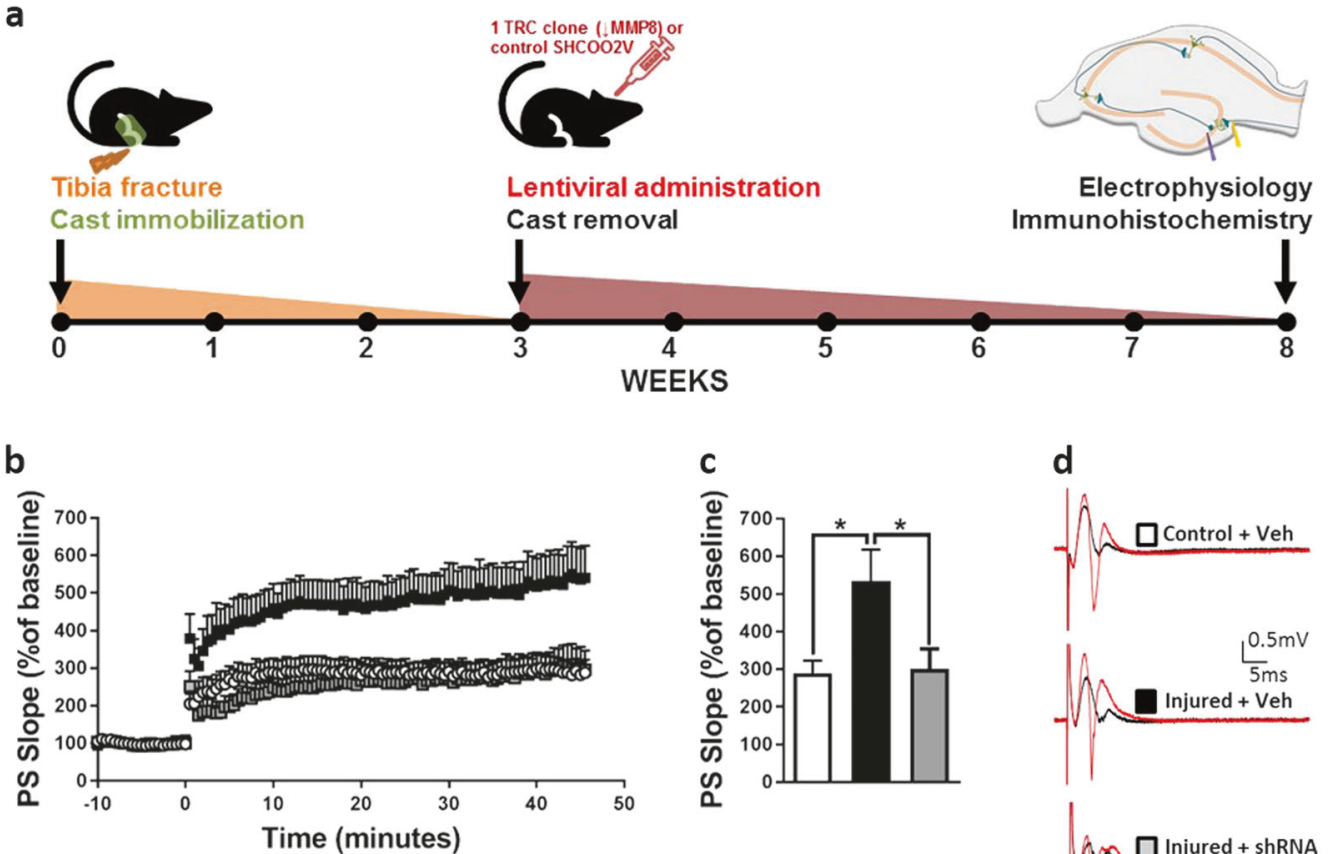

d
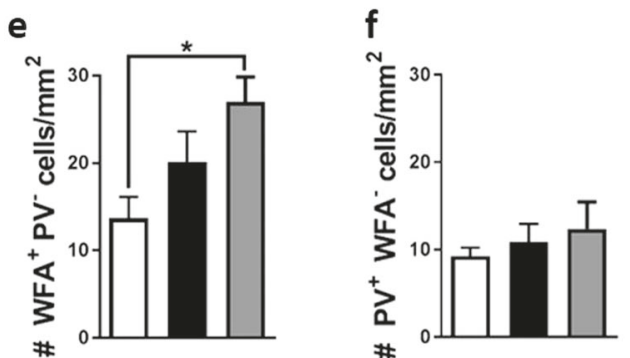

g
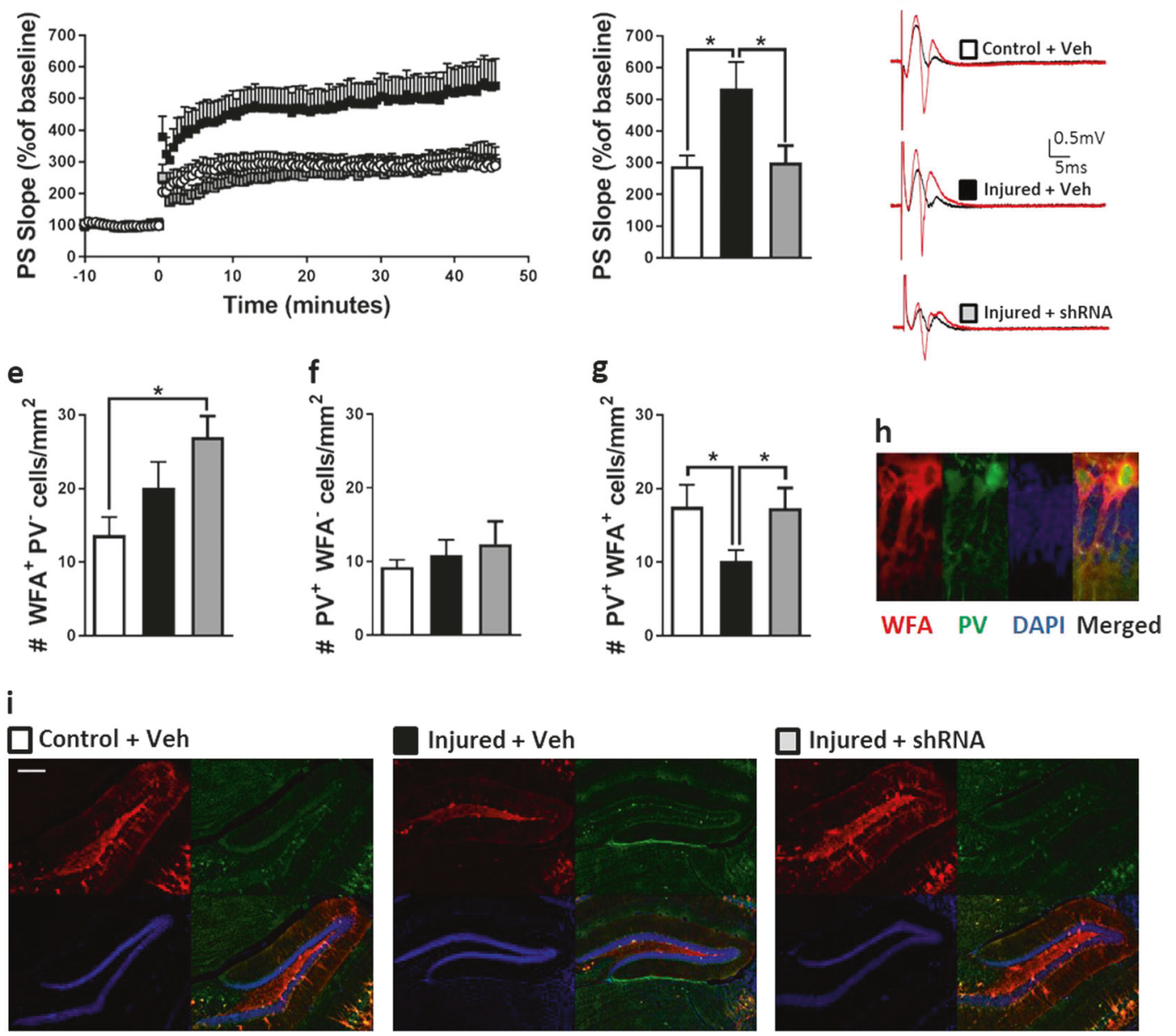

Fig. 4 The stabilization of hippocampal inhibitory interneurons by the perineuronal net is disrupted following peripheral injury. a Experimental timeline: Male C57BL/6 mice underwent tibia fracture and cast immobilization. Three weeks following injury, $\downarrow$ MMP8 or control shRNA lentiviral vector was injected bilaterally in hippocampal formation. Electrophysiological and immunohistochemical analyses were carried out 8 weeks after injury. b-d Injured mice demonstrated increased field EPSP recorded from the DG, which was normalized following MMP8 downregulation (one-way ANOVA, post hoc Holm-Sidak test for multiple comparisons, $n=13$ slices from 4 mice/ group). $\mathbf{e}-\mathbf{i}$ Injury is not linked to a loss of non-parvalbuminergic cells

Acknowledgements The authors thank Dr. Sebastian Alvarado for the thoughtful review of the manuscript and Ms. Ashley M. Hannah for technical assistance. surrounded by a perineuronal net $\left(\mathrm{WFA}^{+} \mathrm{PV}^{-}\right)$or a decrease in the numbers of parvalbumin-positive interneurons that lack a perineuronal net $\left(\mathrm{WFA}^{-} \mathrm{PV}^{+}\right)$. However, immunohistochemical examination showed that injured mice have decreased numbers of inhibitory interneurons that are surrounded by a perineuronal net $\left(\mathrm{WFA}^{+} \mathrm{PV}^{+}\right)$in the DG, suggesting a loss of inhibitory drive in these animals. MMP8 downregulation results in the normalization of $\mathrm{WFA}^{+} \mathrm{PV}^{+}$cell counts (one-way ANOVA, post hoc Holm-Sidak test for multiple comparisons, $n=6-10$ mice/group). Scale bar $=200 \mu \mathrm{m}$. Error bars are s.e.m. $* P<0.05$

Funding The study was supported by the National Institute of Health grant NS072168 to J.D.C. M.T. was supported by the National Institute of Health grant 5T32DA035165-02. 


\section{Compliance with ethical standards}

Conflict of interest The authors declare that they have no conflict of interest.

Open Access This article is licensed under a Creative Commons Attribution 4.0 International License, which permits use, sharing, adaptation, distribution and reproduction in any medium or format, as long as you give appropriate credit to the original author(s) and the source, provide a link to the Creative Commons license, and indicate if changes were made. The images or other third party material in this article are included in the article's Creative Commons license, unless indicated otherwise in a credit line to the material. If material is not included in the article's Creative Commons license and your intended use is not permitted by statutory regulation or exceeds the permitted use, you will need to obtain permission directly from the copyright holder. To view a copy of this license, visit http://creativecommons. org/licenses/by/4.0/

\section{References}

1. McWilliams LA, Goodwin RD, Cox BJ. Depression and anxiety associated with three pain conditions: results from a nationally representative sample. Pain. 2004;111:77-83.

2. Berryman C, Stanton TR, Jane Bowering K, Tabor A, McFarlane A, Lorimer Moseley G. Evidence for working memory deficits in chronic pain: a systematic review and meta-analysis. Pain. 2013;154:1181-96.

3. Tajerian M, Alvarado S, Millecamps M, Vachon P, Crosby C, Bushnell $\mathrm{MC}$, et al. Peripheral nerve injury is associated with chronic, reversible changes in global DNA methylation in the mouse prefrontal cortex. PLoS ONE. 2013;8:e55259.

4. Alvarado S, Tajerian M, Suderman M, Machnes Z, Pierfelice S, Millecamps $M$, et al. An epigenetic hypothesis for the genomic memory of pain. Front Cell Neurosci. 2015;9:88.

5. Massart R, Dymov S, Millecamps M, Suderman M, Gregoire S, Koenigs $\mathrm{K}$, et al. Overlapping signatures of chronic pain in the DNA methylation landscape of prefrontal cortex and peripheral T cells. Sci Rep. 2016;6:19615.

6. Alvarado S, Tajerian M, Millecamps M, Suderman M, Stone LS, Szyf M. Peripheral nerve injury is accompanied by chronic transcriptome-wide changes in the mouse prefrontal cortex. Mol Pain. 2013;9:21.

7. Tajerian M, Leu D, Zou Y, Sahbaie P, Li W, Khan H, et al. Brain neuroplastic changes accompany anxiety and memory deficits in a model of complex regional pain syndrome. Anesthesiology. 2014;121:852-65.

8. Smallwood RF, Laird AR, Ramage AE, Parkinson AL, Lewis J, Clauw DJ, et al. Structural brain anomalies and chronic pain: a quantitative meta-analysis of gray matter volume. J Pain. 2013;14:663-75.

9. Khan SA, Keaser ML, Meiller TF, Seminowicz DA. Altered structure and function in the hippocampus and medial prefrontal cortex in patients with burning mouth syndrome. Pain. 2014;155:1472-80.

10. Mutso AA, Petre B, Huang L, Baliki MN, Torbey S, Herrmann $\mathrm{KM}$, et al. Reorganization of hippocampal functional connectivity with transition to chronic back pain. J Neurophysiol. 2014;111:1065-76.

11. Kalman E, Keay KA. Different patterns of morphological changes in the hippocampus and dentate gyrus accompany the differential expression of disability following nerve injury. $\mathrm{J}$ Anat. 2014;225:591-603.
12. Tajerian M, Sahbaie P, Sun Y, Leu D, Yang HY, Li W, et al. Sex differences in a murine model of complex regional pain syndrome. Neurobiol Learn Mem. 2015;123:100-9.

13. Guo TZ, Offley SC, Boyd EA, Jacobs CR, Kingery WS. Substance $\mathrm{P}$ signaling contributes to the vascular and nociceptive abnormalities observed in a tibial fracture rat model of complex regional pain syndrome type I. Pain. 2004;108:95-107.

14. Terkelsen AJ, Bach FW, Jensen TS. Experimental forearm immobilization in humans induces cold and mechanical hyperalgesia. Anesthesiology. 2008;109:297-307.

15. Chaplan SR, Bach FW, Pogrel JW, Chung JM, Yaksh TL. Quantitative assessment of tactile allodynia in the rat paw. J Neurosci Methods. 1994;53:55-63.

16. Zou Y, Corniola R, Leu D, Khan A, Sahbaie P, Chakraborti A, et al. Extracellular superoxide dismutase is important for hippocampal neurogenesis and preservation of cognitive functions after irradiation. Proc Natl Acad Sci USA. 2012;109:21522-7.

17. De Waele J, Reekmans K, Daans J, Goossens H, Berneman Z, Ponsaerts P. 3D culture of murine neural stem cells on decellularized mouse brain sections. Biomaterials. 2015;41:122-31.

18. Leung VY, Chan WC, Hung SC, Cheung KM, Chan D. Matrix remodeling during intervertebral disc growth and degeneration detected by multichromatic FAST staining. J Histochem Cytochem. 2009;57:249-56.

19. Goncalves JT, Schafer ST, Gage FH. Adult neurogenesis in the hippocampus: from stem cells to behavior. Cell. 2016;167:897-914.

20. Zheng J, Jiang YY, Xu LC, Ma LY, Liu FY, Cui S, et al. Adult hippocampal neurogenesis along the dorsoventral axis contributes differentially to environmental enrichment combined with voluntary exercise in alleviating chronic inflammatory pain in mice. $\mathrm{J}$ Neurosci. 2017;37:4145-57.

21. Liu MG, Chen J. Roles of the hippocampal formation in pain information processing. Neurosci Bull. 2009;25:237-66.

22. Yuste R. From the neuron doctrine to neural networks. Nat Rev Neurosci. 2015;16:487-97.

23. Dityatev A, Schachner M, Sonderegger P. The dual role of the extracellular matrix in synaptic plasticity and homeostasis. Nat Rev Neurosci. 2010;11:735-46.

24. Previtera ML, Langhammer CG, Langrana NA, Firestein BL. Regulation of dendrite arborization by substrate stiffness is mediated by glutamate receptors. Ann Biomed Eng. 2010;38:3733-43.

25. Sur S, Newcomb CJ, Webber MJ, Stupp SI. Tuning supramolecular mechanics to guide neuron development. Biomaterials. 2013;34:4749-57.

26. Ruoslahti E. Brain extracellular matrix. Glycobiology. 1996;6:489-92.

27. McRae PA, Baranov E, Rogers SL, Porter BE. Persistent decrease in multiple components of the perineuronal net following status epilepticus. Eur J Neurosci. 2012;36:3471-82.

28. Vandenbroucke RE, Dejonckheere E, Van Lint P, Demeestere D, Van Wonterghem E, Vanlaere I, et al. Matrix metalloprotease 8dependent extracellular matrix cleavage at the blood-CSF barrier contributes to lethality during systemic inflammatory diseases. J Neurosci. 2012;32:9805-16.

29. Light M, Minor KH, DeWitt P, Jasper KH, Davies SJ. Multiplex array proteomics detects increased MMP-8 in CSF after spinal cord injury. J Neuroinflamm. 2012;9:122.

30. Lee EJ, Han JE, Woo MS, Shin JA, Park EM, Kang JL, et al. Matrix metalloproteinase- 8 plays a pivotal role in neuroinflammation by modulating TNF-alpha activation. J Immunol. 2014;193:2384-93.

31. Han JE, Lee EJ, Moon E, Ryu JH, Choi JW, Kim HS. Matrix metalloproteinase- 8 is a novel pathogenetic factor in focal cerebral ischemia. Mol Neurobiol. 2016;53:231-9. 
32. Murphy G, Willenbrock F. Tissue inhibitors of matrix metalloendopeptidases. Methods Enzymol. 1995;248:496-510.

33. Mroczko B, Groblewska M, Barcikowska M. The role of matrix metalloproteinases and tissue inhibitors of metalloproteinases in the pathophysiology of neurodegeneration: a literature study. J Alzheimers Dis. 2013;37:273-83.

34. Rivera S, Khrestchatisky M, Kaczmarek L, Rosenberg GA, Jaworski DM. Metzincin proteases and their inhibitors: foes or friends in nervous system physiology? J Neurosci. 2010;30:15337-57.

35. Castellano JM, Mosher KI, Abbey RJ, McBride AA, James ML, Berdnik D, et al. Human umbilical cord plasma proteins revitalize hippocampal function in aged mice. Nature. 2017;544:488-92.

36. Chen Y, Chen AQ, Luo XQ, Guo LX, Tang Y, Bao CJ, et al. Hippocampal NR2B-containing NMDA receptors enhance longterm potentiation in rats with chronic visceral pain. Brain Res. 2014;1570:43-53.
37. Wang S, Scott BW, Wojtowicz JM. Heterogenous properties of dentate granule neurons in the adult rat. $J$ Neurobiol. 2000;42:248-57.

38. Deidda G, Allegra M, Cerri C, Naskar S, Bony G, Zunino G, et al. Early depolarizing GABA controls critical-period plasticity in the rat visual cortex. Nat Neurosci. 2015;18:87-96.

39. Chen YJ, Zhang M, Yin DM, Wen L, Ting A, Wang P, et al. ErbB4 in parvalbumin-positive interneurons is critical for neuregulin 1 regulation of long-term potentiation. Proc Natl Acad Sci USA. 2010;107:21818-23.

40. Morishita H, Hensch TK. Critical period revisited: impact on vision. Curr Opin Neurobiol. 2008;18:101-7.

41. Lemons ML, Sandy JD, Anderson DK, Howland DR. Intact aggrecan and chondroitin sulfate-depleted aggrecan core glycoprotein inhibit axon growth in the adult rat spinal cord. Exp Neurol. 2003;184:981-90. 